\title{
Condições de biossegurança em estabelecimentos de atendimento médico-veterinário no município de Ribeirão Preto, SP
}

Biosafety in veterinarian establishments in the city of Ribeirão Preto, SP, Brazil

\section{Condiciones de bioseguridad en los establecimientos de asistencia veterinaria en la ciudad de Ribeirão Preto, SP, Brasil}

\section{Dario Valente; ${ }^{1}$ Carlos Alberto d'Ávilla de Oliveira; ${ }^{2}$ Vânia Cantarela Rodrigues; ${ }^{3}$ Hélio Trebbi ${ }^{4}$}

Divisão de Vigilância Sanitária da Secretaria Municipal da Saúde de Ribeirão Preto. Ribeirão Preto, SP, Brasil 
VALENTE, D. et al. Biossegurança em estabelecimentos veterinários. / Biosafety in veterinarian establishements. / Bioseguridad en establecimientos veterinarios. Rev. Educ. Contin. CRMV-SP/Contin. Educ. J. CRMV-SP, São Paulo, v. 7, n. 1/3, p. 45-54, 2004.

\section{Resumo}

Objetivo: Detectar, nos estabelecimentos que prestam atendimento médico-veterinário, condições específicas que podem comprometer a biossegurança, a saber: a) utilização de abrigo adequado de resíduos; b) adequação às normas de proteção radiológica; c) uso de equipamentos de proteção individual (EPIs); d) vacinação pré-exposicional contra raiva e tétano das pessoas sob risco; e) utilização de geladeira exclusiva para armazenamento de produtos biológicos; f) emprego de termômetro de máxima e mínima; g) uso de coletor adequado de material. Material e Método: Estudo retrospectivo cujos dados foram obtidos dos relatórios de inspeção sanitária constantes nos processos de solicitação de Licença de Funcionamento à Vigilância Sanitária da Secretaria Municipal da Saúde de Ribeirão Preto, SP. Foram avaliados os roteiros de inspeção de 36 estabelecimentos veterinários (27 clínicas e nove consultórios), inspecionados de 18 de setembro de 2000 a 5 de setembro de 2001. Resultados: Dos 36 estabelecimentos, oito $(22,2 \%)$ possuíam abrigo adequado para resíduos de serviços de saúde; sete $(19,4 \%)$ dispunham de aparelho de raios X, mas somente um (14,3\%) atendia à legislação relativa às normas de proteção radiológica; $24(66,7 \%)$ não tinham esquema de vacinação pré-exposicional contra raiva e tétano para todos os funcionários em contato com cães e gatos; em 34 (94\%) utilizavam-se equipamentos de proteção, embora, na maioria das vezes, se limitassem a focinheiras; em $13(36,1 \%)$ não havia geladeira exclusiva para armazenar produtos biológicos - alimentos, água e principalmente vacinas estavam armazenados juntos; em $21(58,3 \%)$ não se monitorava a temperatura dos produtos biológicos com termômetro de máxima e mínima; em $26(72,2 \%)$ o coletor utilizado para materiais perfurocortantes não era adequado. Conclusões: Os dados obtidos permitem concluir que os estabelecimentos veterinários de Ribeirão Preto não apresentam condições adequadas de biossegurança, o que requer intervenção das autoridades sanitárias em relação aos responsáveis técnicos pelos estabelecimentos, visando corrigir as irregularidades encontradas.

Palavras-chave: Vigilância sanitária. Registros, utilização. Hospitais veterinários. Consultórios médicos. Disposição de resíduos de saúde. Proteção radiológica. Exposição ocupacional. Produtos biológicos, provisão e distribuição.

\section{Introdução}

A biossegurança deixou de ser preocupação para tornar-se exigência. Num estabelecimento veterinário (EV), os cuidados devem começar pelas instalações, escolha de equipamentos, treinamento e imunização de toda a equipe envolvida no atendimento.

Biossegurança e qualidade estão vinculadas pelo conceito de boas práticas, entendido como um conjunto de ações que permite alcançar um grau satisfatório de segurança diante dos riscos a que estão expostos os profissionais dos serviços, os clientes e o meio ambiente. Portanto, biossegurança também é problema de qualidade. ${ }^{1}$

Não é difícil encontrar profissionais da área da saúde, inclusive de algumas áreas específicas da medicina veterinária, que não valorizam as medidas de proteção - individuais e coletivas - de eficácia amplamente comprovada. ${ }^{1}$

A biossegurança pode ser definida como o conjunto de ações voltadas para a prevenção, minimização ou eliminação de riscos inerentes às atividades que possam comprometer a saúde humana, dos animais e do meio ambiente, ou a qualidade dos trabalhos realizados. ${ }^{2}$ Pode ainda ser definida como "segurança no manejo de produtos e técnicas biológicas". 2

Etimologicamente, a palavra biossegurança significa vida + segurança ${ }^{3}$, podendo ser entendida, em sentido amplo, como o conjunto das medidas necessárias para uma vida livre de perigos. ${ }^{1}$ Assim, normas de biossegurança englobam todas as medidas 
VALENTE, D. et al. Biossegurança em estabelecimentos veterinários. / Biosafety in veterinarian establishements. / Bioseguridad en establecimientos veterinarios. Rev. Educ. Contin. CRMV-SP/Contin. Educ. J. CRMV-SP, São Paulo, v. 7, n. 1/3, p. 45-54, 2004.

que visam evitar riscos físicos (radiação, temperatura, ruídos, iluminação, etc.), ergonômicos (posturais), químicos (substâncias tóxicas), biológicos (agentes infecciosos), psicológicos (estresse) $)^{1}$, mecânicos (mordeduras) e operacionais. ${ }^{4}$

Tanto o exagero quanto o desprezo às medidas de biossegurança devem ser evitados. Em razão da evolução tecnológica, são extremamente necessários o aprimoramento e a divulgação das medidas de biossegurança para médicos veterinários. ${ }^{1}$

À medida que a humanidade evoluiu, o maior conhecimento sobre agentes etiológicos e fatores envolvidos na suscetibilidade individual têm permitido, de forma mais racional, o estabelecimento de medidas que reduzem o risco de aquisição e propagação de várias doenças, com especial destaque para as infectocontagiosas. ${ }^{1}$

Para implementar medidas de biossegurança num determinado ambiente, a tomada de decisões deve fundamentar-se em três conceitos básicos: ${ }^{4}$

- reconhecimento dos riscos;

- avaliação dos riscos;

- controle dos riscos.

No campo da biossegurança, as primeiras investidas concretas surgiram em 1976, quando o National Institute of Health (NIH) dos Estados Unidos divulgou normas de segurança laboratorial que deveriam ser obrigatoriamente seguidas pelos laboratórios que estavam recebendo verbas federais. ${ }^{2}$

No Brasil, as discussões sobre o assunto ocorrem de modo isolado e normalmente sem correlação com a expressão biossegurança, principalmente nas áreas relacionadas à medicina veterinária. Uma das poucas normas técnicas de biossegurança aplicáveis aos estabelecimentos veterinários é a Portaria SVS n. 453/1998, ${ }^{5}$ que normatiza as medidas de proteção radiológica. ${ }^{6}$ Para evitar os graves efeitos biológicos deletérios resultantes do emprego inadequado de radiação ionizante, ${ }^{6}$. são indispensáveis a utilização de equipamentos de proteção individual
(EPIs) e a eficiência da blindagem do cabeçote do tubo de raios $\mathrm{X}$, das paredes, das portas e dos visores plumbíferos - avaliada por levantamento radiométrico e testes de radiação de fuga do aparelho de raios X. Em muitas áreas da medicina veterinária, alguns assuntos exibem sérias implicações relacionadas à biossegurança, por exemplo: a destinação de resíduos infectantes e de material perfurocortante; a conservação correta de produtos biológicos; o controle adequado de medicamentos entorpecentes ou psicoativos; a utilização de quimioterápicos $;^{7}$ o despejo, diretamente na rede de esgoto, de pêlos oriundos de banho; a utilização de EPIs durante a aplicação de técnicas de contenção ${ }^{8}$ técnicas de esterilização; vacinação pré-exposicional contra raiva e tétano dos profissionais que mantêm contato direto com cães e gatos; a estrutura física das diversas dependềncias do estabelecimento veterinário.

A importância do tema e a carência de trabalhos sobre biossegurança e medicina veterinária estimularam a realização deste trabalho. Seu objetivo foi detectar, nos estabelecimentos que prestam atendimento médico-veterinário, condições específicas que podem comprometer a biossegurança, a saber: a) utilização de abrigo adequado de resíduos; b) adequação às normas de proteção radiológica; c) uso de equipamentos de proteção individual (EPIs); d) vacinação pré-exposicional contra raiva e tétano das pessoas sob risco; e) utilização de geladeira exclusiva para armazenamento de produtos biológicos; f) emprego de termômetro de máxima e mínima; g) uso de coletor adequado de material.

\section{Material e Método}

Realizou-se um estudo retrospectivo cujas informações foram obtidas dos relatórios de inspeção sanitária constantes nos processos de solicitação de Licença de Funcionamento (LF) da Vigilância Sanitária da Secretaria Municipal da Saúde (Visa-SMS) de Ribeirão Preto, SP. Todos os EVs que prestam atendimento médico-veterinário (hospitais, clínicas 
e consultórios veterinários) necessitam de cadastro e/ou LF concedida pela Visa-SMS. A LF é liberada somente quando se verifica, em inspeção sanitária, que o EV atende às exigências mínimas para funcionamento.

Foram avaliados os roteiros de inspeção de 36 EVs, 27 clínicas e nove consultórios veterinários, inspecionados de 18 de setembro de 2000 a 5 de setembro de 2001. Durante as inspeções, preencheuse um roteiro específico para verificação das condições de biossegurança (Anexo 1).

As inspeções sanitárias foram realizadas por uma mesma fiscal sanitária visando à padronização dos critérios adotados durante as inspeções, sempre supervisionadas por um médico veterinário.

\section{Resultados}

Dos 36 estabelecimentos veterinários (EV) inspecionados, oito (22,2\%) tinham abrigos de resíduos de serviços de saúde adequados, e 28 deles $(77,8 \%)$ apresentavam-se inadequados (Tabela 1).

Tabela 1 - Adequação dos estabelecimentos veterinários segundo a condição do abrigo de resíduos de saúde em Ribeirão Preto, SP, no período de 18 de setembro de 2000 a 5 de setembro de 2001

\begin{tabular}{|ccc|}
\hline $\begin{array}{c}\text { Abrigo de } \\
\text { resíduos de saúde }\end{array}$ & \multicolumn{2}{c|}{$\begin{array}{c}\text { Estabelecimento } \\
\text { veterinário }\end{array}$} \\
\cline { 2 - 3 } & $\mathrm{N}$ & $\%$ \\
\hline Adequada & 8 & 22,2 \\
Inadequada & 28 & 77,8 \\
\hline Total & 36 & $\mathbf{1 0 0 , 0}$ \\
\hline
\end{tabular}

Em relação ao emprego de radiação ionizante (Tabela 2), sete $(19,4 \%)$ EVs possuíam aparelho de raios, e somente um $(14,3 \%)$ atendia à legislação relativa às normas de proteção radiológica.

Equipamentos de proteção (EP) eram utilizados em 34 (94,4\%) EVs, enquanto em dois $(5,6 \%)$ não se constatou utilização de EPs (Tabela 3).
Tabela 2 - Adequação dos estabelecimentos veterinários em relação às normas de proteção radiológica, em Ribeirão Preto, SP, no período de 18 de setembro de 2000 a 5 de setembro de 2001

\begin{tabular}{|ccc|}
\hline $\begin{array}{c}\text { Proteção } \\
\text { radiológica }\end{array}$ & \multicolumn{2}{c|}{$\begin{array}{c}\text { Estabelecimento } \\
\text { veterinário }\end{array}$} \\
\cline { 2 - 3 } & $\mathbf{N}$ & $\%$ \\
\hline Adequada & 1 & 14,3 \\
Inadequada & 6 & 85,7 \\
\hline Total & 7 & 100,0 \\
\hline
\end{tabular}

Tabela 3 - Distribuição dos estabelecimentos veterinários em relação à utilização de equipamentos de proteção, em Ribeirão Preto, SP, no período de 18 de setembro de 2000 a 5 de setembro de 2001

\begin{tabular}{|ccc|}
\hline $\begin{array}{c}\text { Equipamento de } \\
\text { proteção }\end{array}$ & \multicolumn{2}{c|}{$\begin{array}{c}\text { Estabelecimento } \\
\text { veterinário }\end{array}$} \\
\cline { 2 - 3 } & $\mathrm{N}$ & $\%$ \\
\hline Utilizava & 34 & 94,4 \\
Não utilizava & 2 & 5,6 \\
\hline Total & 36 & $\mathbf{1 0 0 , 0}$ \\
\hline
\end{tabular}

Em relação ao esquema de vacinação préexposicional contra raiva e tétano (Tabela 4), em 12 $(33,3 \%)$ EVs todos os funcionários em contato direto com cães e gatos estavam adequadamente vacinados, enquanto em $24(66,7 \%)$ foram encontrados funcionários sem proteção vacinal.

Havia geladeira exclusiva para produtos biológicos em $23(63,9 \%)$ EVs, e em $13(36,1 \%)$ não (Tabela 5).

Tabela 4 - Adequação dos estabelecimentos veterinários ao esquema de vacinação pré-exposicional contra raiva e tétano, em Ribeirão Preto, SP, no período de 18 de setembro de 2000 a 5 de setembro de 2001

\begin{tabular}{|ccc|}
\hline $\begin{array}{c}\text { Esquema de vacinação } \\
\text { pré-exposicional contra } \\
\text { raiva e tétano }\end{array}$ & $\begin{array}{c}\text { Estabelecimento } \\
\text { veterinário }\end{array}$ \\
\cline { 2 - 3 } & $\mathrm{N}$ & $\%$ \\
\hline Adequado & 12 & 33,3 \\
\hline Inadequado & 24 & 66,7 \\
\hline Total & 36 & $\mathbf{1 0 0 , 0}$ \\
\hline
\end{tabular}


VALENTE, D. et al. Biossegurança em estabelecimentos veterinários. / Biosafety in veterinarian establishements. / Bioseguridad en establecimientos veterinarios. Rev. Educ. Contin. CRMV-SP/Contin. Educ. J. CRMV-SP, São Paulo, v. 7, n. 1/3, p. 45-54, 2004.

Tabela 5-Distribuição dos estabelecimentos veterinários em relação à utilização de geladeira exclusiva para produtos biológicos, em Ribeirão Preto, SP, no período de 18 de setembro de 2000 a 5 de setembro de 2001

\begin{tabular}{|ccc|}
\hline $\begin{array}{c}\text { Geladeira esclusiva } \\
\text { para produto biológico }\end{array}$ & \multicolumn{2}{c|}{$\begin{array}{c}\text { Estabelecimento } \\
\text { veterinário }\end{array}$} \\
\cline { 2 - 3 } & $\mathrm{N}$ & $\%$ \\
\hline Adequada & 23 & 63,9 \\
\hline Inadequada & 13 & 36,1 \\
\hline Total & 36 & $\mathbf{1 0 0 , 0}$ \\
\hline
\end{tabular}

Em relação ao termômetro de máxima e mínima para monitoramento da temperatura de produtos biológicos (Tabela 6), em 15 (41,7\%) EVs o termômetro era utilizado adequadamente, enquanto em 21 $(58,3 \%)$ não havia o instrumento ou sua utilização estava incorreta.

Em $26(72,2 \%)$ EVs não existia coletor adequado de material perfurocortante, enquanto em dez $(27,8 \%)$ os coletores eram adequados (Tabela 7$)$.

Tabela 6 - Distribuição dos estabelecimentos veterinários em relação à utilização de termômetro de temperaturas máxima e mínima na geladeira de produtos biológicos, em Ribeirão Preto, SP, no período de 18 de setembro de 2000 a 5 de setembro de 2001

\begin{tabular}{|ccr|}
\hline $\begin{array}{c}\text { Utilização de termômetro } \\
\text { de máxima e mínima }\end{array}$ & \multicolumn{2}{c|}{$\begin{array}{c}\text { Estabelecimento } \\
\text { veterinário }\end{array}$} \\
\cline { 2 - 3 } & $\mathrm{N}$ & $\%$ \\
\hline Adequada & 15 & 41,7 \\
\hline Inadequada & 21 & 58,3 \\
\hline Total & 36 & 100,0 \\
\hline
\end{tabular}

Tabela 7 - Distribuição dos estabelecimentos veterinários em relação à utilização de coletores de material perfurocortante, em Ribeirão Preto, SP, no período de 18 de setembro de 2000 a 5 de setembro de 2001

\begin{tabular}{|ccc|}
\hline $\begin{array}{c}\text { Coletor de material } \\
\text { perfurocortante }\end{array}$ & \multicolumn{2}{c|}{$\begin{array}{c}\text { Estabelecimento } \\
\text { veterinário }\end{array}$} \\
\cline { 2 - 3 } & $\mathrm{N}$ & $\%$ \\
\hline Adequado & 10 & 27,8 \\
\hline Inadequado & 26 & 72,2 \\
\hline Total & 36 & 100,0 \\
\hline
\end{tabular}

\section{Discussão}

\section{Considerações Gerais}

Constatou-se que as condições dos estabelecimentos de atendimento médico-veterinário (EVs) de Ribeirão Preto, no momento da inspeção, geraram a possibilidade de comparar extremos. Refletem, portanto, a situação encontrada no momento da inspeção.

O roteiro constante no Anexo 1, utilizado para orientar o procedimento, demonstrou ser bastante útil para padronizar a inspeção sanitária e registrar as condições dos EVs.

As condições de biossegurança abordadas no presente estudo constituem alguns dos itens avaliados durante a inspeção; os outros serão tema de trabalho posterior.

\section{Os Estabelecimentos Veterinários de Ribeirão Preto}

Em sua maioria, os EVs de Ribeirão Preto $(77,8 \%)$ foram considerados inadequados em relação à existência de abrigo de resíduos de saúde. Os EVs não tinham tal recurso, ou este não estava adequado. Os EVs devem ter abrigo de resíduos gerados no local, construído com material de fácil higienização, com separação dos resíduos infectantes e com capacidade para armazenar o triplo do volume gerado durante o período de coleta. As condições encontradas implicam em graves conseqüências para o meio ambiente e a segurança das pessoas que trabalham no local, além de favorecerem a proliferação de roedores, insetos e outros artrópodes.

Dos EVs inspecionados, somente sete $(19,4 \%)$ dispunham de aparelho de raios X; destes, apenas um $(14,3 \%)$ estava adequado em relação às normas de proteção radiológica. As principais irregularidades foram: a não-apresentação de laudos radiométricos (atestados por um físico); a não-implantação de pro- 
grama de garantia de qualidade; a não-utilização de dosímetro individual, avental de chumbo, protetor de tireóide, luvas e óculos plumbíferos; falta de lâmpada de segurança e símbolo de radiação na porta da sala de raios X. Um dos graves problemas resultantes das falhas de radioproteção é a manifestação tardia dos efeitos deletérios da radiação, por exemplo, o aumento da probabilidade da ocorrência de câncer. ${ }^{6}$

Foram avaliados dois aspectos dos EPs: a utilização de equipamentos diretamente pelos profissionais, os chamados EPIs (luvas, óculos e avental), e o uso de equipamentos diretamente para os animais (focinheira, gaiola de contenção e cambão). Em dois $(5,6 \%)$ dos EVs não se utilizava qualquer tipo de EP, enquanto em 34 (94,4\%) usava-se pelo menos um. O emprego de EPs assume importância especial nos EVs de pequenos animais. A inexistência e a falta de uso desses equipamentos favorecem muito a ocorrência de acidentes, que podem ser graves, em conseqüência das características de alguns animais (gatos e cães agressivos).

Em função do contato direto dos profissionais (veterinários e auxiliares) com os animais durante o atendimento, é grande o risco da ocorrência de pequenos acidentes - pequenas arranhaduras ou mordeduras, por exemplo - o que torna indispensável a vacinação pré-exposicional contra raiva e tétano. Particularmente preocupante foi o achado de que em $24(66,7 \%)$ dos EVs a vacinação pré-exposicional não era realizada ou não era feita corretamente, ou seja, em serviço público, com acompanhamento por profissionais especializados e realização de exames de titulação de anticorpos.

É particularmente importante o alto percentual de inadequação - 36,1\% (13 EVs) - em relação ao uso de geladeira exclusiva para armazenamento de produtos biológicos, que permite o controle adequado da temperatura. A alta freqüência com que se abre uma geladeira que armazena alimentos e líquidos (água e refrigerantes) dificulta a manutenção da temperatura na faixa adequada (de 2 a $7^{\circ} \mathrm{C}$ ), além de aumentar a probabilidade de contaminação dos alimentos.

Uma das exigências para conservar bem os produtos biológicos é o monitoramento permanente das temperaturas máxima e mínima no interior dos equipamentos de refrigeração; para que seja eficiente, é indispensável o uso correto de um termômetro de máxima e mínima. Chama a atenção o fato de que na maioria dos EVs - 21 (58,3\%) - não existia termômetro de máxima e mínima ou desconhecia-se seu uso correto.

Somente em dez $(27,8 \%)$ EVs foi constatado o uso de coletores adequados de material perfurocortante, que previnem acidentes com as pessoas que manipulam esse material - funcionários do EV e trabalhadores da coleta e transporte dos resíduos. As paredes dos coletores devem ser rígidas e sua abertura de tamanho adequado. Algumas das irregularidades verificadas foram o emprego de garrafas plásticas com abertura tão reduzida que dificultava o descarte do material, e o uso de coletores grandes, que demoravam muito para encher, permanecendo muito tempo no estabelecimento.

\section{Conclusões}

Os dados obtidos permitem concluir que, em sua maioria, os EVs de Ribeirão Preto não apresentam condições adequadas de biossegurança, o que exige rigorosa intervenção das autoridades sanitárias em relação aos responsáveis técnicos dos estabelecimentos, visando corrigir as irregularidades encontradas. 
VALENTE, D. et al. Biossegurança em estabelecimentos veterinários. / Biosafety in veterinarian establishements. / Bioseguridad en establecimientos veterinarios. Rev. Educ. Contin. CRMV-SP/Contin. Educ. J. CRMV-SP, São Paulo, v. 7, n. 1/3, p. 45-54, 2004.

\section{Questionário para verificação das condições de biossegurança em estabelecimentos veterinários:}

Data:

Classificação:

Nome (Fantasia):

Razão Social:

Endereço:

Bairro:

Ribeirão Preto, SP - CEP:

Licença de Funcionamento:

Inscrição do Estabelecimento no CRMV/SP n².

Responsável Técnico: Dr. (a):

CRMV/SP n:

1. Das instalações:

\subsection{Abrigo para resíduos}

\begin{tabular}{|l|l|}
\hline a. Possui abrigo para resíduos: & SIM 2. NÃO \\
\hline b. Separado para resíduo comum e contaminado: & \\
\hline c. Dimensionado para abrigar o triplo do volume gerado durante o período de coleta: & \\
\hline d. Constituído de material de fácil higienização: & \\
\hline
\end{tabular}

\subsection{Salas de recepção e atendimento (espera/consulta/coleta/cirurgia)}

a. Adequadas ao Decreto Estadual $\mathrm{n}^{2} 40.400 / 1995$ :

\subsection{Sala de esterilização}

a. Possui sala de esterilização:

Área total: .................. $\mathrm{m}^{2}$

Menor dimensão no plano horizontal:

$\mathrm{m}$

b. Piso - liso, impermeável e resistente a pisoteio e desinfetantes:

c. Paredes - impermeabilizadas até o teto:

d. Provida de equipamento de esterilização seca (estufa):

e. Provida de equipamento de esterilização úmida (autoclave):

\subsection{Sala para abrigo de animais}

a. Acesso afastado das dependências destinadas a cirurgias e laboratórios:

b. Piso - liso, impermeável e resistente a pisoteio e desinfetantes:

c. Paredes - impermeabilizadas até altura de $2 \mathrm{~m}$ :

d. Provida de instalações necessárias ao conforto e segurança dos animais:

e. Propicia ao pessoal que nela trabalha condições adequadas de higiene e segurança ao desempenho de suas funções:

f. Suas dimensões são compatíveis com as espécies a que se destinam:

g. Provida de dispositivos que evitem a propagação de ruídos incômodos e exalação de odores:

h. Provida de água corrente suficiente para higienização do ambiente:

i. O escoamento de águas servidas é ligado diretamente à rede de esgoto ou à fossa séptica com poço absorvente:

j. Portas e janelas providas de telas:

k. O destino das fezes é adequado: 
VALENTE, D. et al. Biossegurança em estabelecimentos veterinários. / Biosafety in veterinarian establishements. / Bioseguridad en establecimientos veterinarios. Rev. Educ. Contin. CRMV-SP/Contin. Educ. J. CRMV-SP, São Paulo, v. 7, n. 1/3, p. 45-54, 2004.

\subsection{Sala de radiografias}

1. SIM 2. NÃO

a. Possui equipamento de raios $\mathrm{X}$ :

b. Possui sala de radiografias:

c. Suas dimensões são compatíveis com as espécies a que se destinam:

d. Possui laudo radiométrico de acordo com a legislação vigente:

e. É um estabelecimento comercial ou industrial:

f. Possui aparelho radiológico portátil com alvará específico:

\subsection{Salas de banho e tosa}

a. Piso - liso, impermeável e resistente a pisoteio e desinfetantes:

b. Paredes - impermeabilizadas até altura de $2 \mathrm{~m}$ :

c. A banheira possui paredes lisas e impermeáveis:

d. O escoamento de águas servidas é ligado diretamente ao esgoto:

e. O escoamento de água da banheira possui caixa de sedimentação:

1. SIM 2. NÃO

\section{Das condições de funcionamento e pessoal:}

a. Possui médico veterinário durante o período de atendimento:

1. SIM 2. NÃO

b. Possui auxiliar de veterinário:

c. Possui coleta especial para resíduos da área de saúde:

d. Utiliza EPIs durante os atendimentos (focinheiras, luvas, cambões, gaiolas de contenção, etc.):

e. Todas as pessoas que mantêm contato direto com animais (cães e gatos) possuem vacinação pré-exposicional contra raiva e vacina contra tétano:

f. Os produtos biológicos são armazenados em geladeiras exclusivas:

g. A geladeira de produtos biológicos possui termômetro de máxima e mínima:

h. Possui exalação de odores indesejáveis:

i. Apresenta ruídos incômodos:

j. Apresenta proliferação de insetos, roedores e artrópodes nocivos:

k. Utiliza coletor adequado para material perfurocortante:

\section{Da localização:}

a. Localiza-se em zona urbana:

b. Localiza-se em zona estritamente residencial:

\section{Do uso de drogas especiais:}

a. O estabelecimento utiliza drogas sob controle especial (anestésicos, tranqüilizantes, etc.):

b. O estabelecimento possui livro de controle de drogas especiais:

\section{Observações:}

\section{Responsável pela inspeção:}


VALENTE, D. et al. Biossegurança em estabelecimentos veterinários. / Biosafety in veterinarian establishements. / Bioseguridad en establecimientos veterinarios. Rev. Educ. Contin. CRMV-SP/Contin. Educ. J. CRMV-SP, São Paulo, v. 7, n. 1/3, p. 45-54, 2004.

\section{Abstract}

Objective: To identify specific conditions in veterinary establishments that may jeopardize biosafety, such as: a) proper disposal of medical waste; b) compliance with radiological safety rules; c) use of personal protection equipment; d) pre-exposure vaccination of directly involved personnel against rabies and tetanus; $e$ ) utilization of a refrigerator exclusive for storage of biological products; $f$ ) use of Maximum-Minimum thermometer; $g$ ) employment of adequate disposal container. Material and Method: Retrospective study based on data collected from sanitary inspection reports concerning requests for Operation License, filed within the Sanitary Surveillance section of the Municipal Health Secretariat of Ribeirão Preto, SP, Brazil. The files of 36 veterinary establishments inspected between September-18-2000 and September-5-2001, were evaluated. Results: Among the 36 establishments evaluated, 8 (22.2\%) disposed of medical waste properly; 7 (19.4\%) had X-ray equipment, but only 1 (14.3\%) met the legal requirements concerning radiological protection; 24 (66.7\%) failed to provide pre-exposure vaccination against rabies and tetanus for all workers handling dogs and cats; personal protection equipment was used in 34 (94\%), but was mostly limited to muzzles; in 13 (36.1\%) the refrigerator was not exclusive for storage of biological products - food, water and biological products, especially vaccines, were stored together; in 21 (58.3\%) a Maximum-Minimum thermometer was not used for temperature control of biological products and $26(72.2 \%)$ did not have adequate sharps disposal container. Conclusions: Based on the data evaluated, it was concluded that most veterinary establishments in Ribeirão Preto do not offer satisfactory biosafety conditions and that the sanitary authorities must act against the license holders, to ensure that such irregularities be corrected.

Keywords: Health surveillance. Records, utilization. Hospitals, animals. Physician's offices. Medical waste disposal. Radiation protection. Occupational exposure. Biological products, supply and distribution.

\section{Resumen}

Objetivo: Descubrir en los establecimientos que prestan atención médico-veterinaria, condiciones específicas que pueden poner en riesgo la bioseguridad, como: a) utilización de lugares adecuados para residuos sanitarios; b) adecuación a las normas de protección radiológica; c) uso de equipos de protección individual (EPI's); d) vacunación de preexposición contra la rabia y tétanos de las personas con riesgo; e) uso de nevera exclusiva para el almacenamiento de productos biológicos; f) uso de termómetro de temperatura máxima y mínima; y g) uso de colector adecuado de material. Material y Método: Estudio retrospectivo. Los datos se obtuvieron de los informes realizados por la Vigilancia Sanitaria de la Secretaría Municipal de Salud de Ribeirão Preto, SP, Brasil, en el momento de recibir de los establecimientos la solicitud de Licencia de Funcionamiento. Se analizaron las inspecciones de 36 establecimientos veterinarios (27 clínicas y 9 consultorios), ejecutadas entre el 18 de septiembre de 2000 y el 5 de septiembre de 2001. Resultados: De los 36 establecimientos, 8 (el 22,2\%) poseían un lugar adecuado para residuos de productos sanitarios; 7 (el 19,4\%) poseían aparato de rayos X, aunque solamente 1 (el 14,3\%) respondia a la legislación relativa a las normas de protección radiológica; 24 (el 66,7\%) no contaban con un esquema de vacunación de preexposición contra la rabia y tétanos, para aquellos funcionarios que entrasen en contacto con perros y gatos; en 34 (94\%) se utilizaban equipos de protección, aunque en la mayoría de las veces se limitasen a bozal; en 13 (el $36,1 \%)$ no había una nevera exclusiva para almacenar productos biológicos (alimentos, agua y otros, se almacenaban con vacunas); en 21 (el 58,3\%) no se controlaba la temperatura de los productos biológicos con termómetro de temperatura máxima y mínima; en 26 (el 72,2\%) el colector para materiales cortopunzantes, no era adecuado. Conclusiones: Los datos obtenidos permiten concluir que los establecimientos veterinarios de Ribeirão Preto no ofrecen las condiciones de bioseguridad adecuadas, lo cual hace necesaria la intervención de las autoridades sanitarias para que junto a los responsables técnicos de dichos establecimientos se corrijan de inmediato las irregularidades que se encontraron.

Palabras-clave: Vigilancia sanitaria. Registros, utilización. Hospitales veterinarios. Consultorios médicos. Disposición de residuos médicos. Protección radiológica. Exposición ocupacional. Productos biológicos, provisión y distribución. 


\section{Referências}

1. CAVAlCANTE, N. J. F.; BARBIERI, D. D.; MONTEIRO, A. L. C. Biossegurança. Atualidades em DST/AIDS, v. 1, n. 1, 1998.

2. SANT'ANA, A. Biossegurança no Brasil: a necessidade de uma política consistente. In: Teixeira, P.; Valle, S. (Org.). Biossegurança: uma abordagem multidisciplinar. Rio de Janeiro: Editora FIOCRUZ, 1996. p. 15-25

3. FERREIRA, A. B. H. Dicionário Aurélio básico da língua portuguesa. São Paulo: Nova Fronteira, 1994-1995.

4. BRASIL. Ministério da Saúde. Segurança no ambiente hospitalar. Brasília, DF: Ministério da Saúde, 1995. 196 p.
5. BRASIL. Ministério da Saúde. Secretaria de Vigilância Sanitária. Diretrizes de proteção radiológica em radiodiagnóstico: Portaria SVS n² 453/1998. Brasília, DF: Ministério da Saúde, 1998.

6. PROTEÇÃO radiológica. Clínica Veterinária, v. 1, n. 5 , p. $20,1996$.

7. NORMAS de segurança para o uso de vincristina no tratamento de tumor venéreo transmissível em cães. Clínica Veterinária, v. 4, n. 18, p. 32-33, 1999.

8. FAÇA fácil, dardos e zarabatanas. Clínica Veterinária, v. 5, n. 28, p. 55-60, 2000. 